\title{
> Cinco ensaios de The Philosophy of Style, de Herbert Spencer
}

> Five essays from Herbert Spencer's The Philosophy of Style translated to portuguese

\section{por Thaís Fernandes dos Santos}

Pós-graduanda em Letras (Língua e Literatura Inglesas) pela Universidade Estácio de Sá (UNESA). E-mail: thaisf.santos@bol.com.br. ORCID: 0000-0003-0502-5832. 
The words of a language often reflect not so much the reality

of the world, but the interests of the people who speak it. ${ }^{1}$

\section{Apresentação}

Herbert Spencer, nascido em Derbyshire, Inglaterra, em 27 de abril de 1820, e falecido em Brighton, Sussex, em 8 de dezembro de 1903, foi um proeminente escritor e um dos maiores intelectuais europeus do movimento positivista do século XIX. Filósofo, sociólogo, biólogo, educador e cientista, fortemente influenciado pela teoria da evolução de Charles Darwin, também se dedicou ao jornalismo e atuou como editor do The Economist.

Spencer era o mais velho de nove filhos e o único a sobreviver da união entre o professor e matemático William George Spencer e Harriet Spencer. E, curiosamente, ele não recebeu educação formal, porém, desde muito jovem, desenvolveu hábitos de estudos, o que lhe despertou interesse pelas ciências exatas e evolução natural.

Polímata, cujos trabalhos contribuíram para diversas áreas de conhecimento, como antropologia, economia, ética, religião, psicologia, além da sociologia, filosofia e literatura; entretanto, Spencer foi autor de prolíficas obras filosóficas e educativas, incluindo A Educação Intelectual, Moral e Física (1863) e Os Princípios da Sociologia (1874), e revelou-se um defensor do estudo científico, sobretudo um renomado filósofo da sociedade vitoriana.

\subsection{A obra The Philosophy of Style. breves comentários}

O livro intitulado The Philosophy of Style, publicado originalmente na Inglaterra, em 1852, é composto por duas partes.

\footnotetext{
${ }^{1}$ Robert Frank Palmer, Semantics: A new outline, 1976, p. 21.
} 
Da primeira parte, apresentam-se ao leitor as traduções de dois ensaios: Economy in the Use of Words e The Effect of Poetry Explained, os quais destacamse pela descrição sobre a aplicação da economia às palavras, ao texto escrito; informativos, ainda, da prosa criativa, observando três aspectos: transposição, o uso de figuras de linguagem e a questão da brevidade, especialmente no uso de interjeições. Já a segunda parte constitui um conjunto de quatro ensaios: Explanation of Climax, Antithesis and Anticlimax, Need of Variety e The Ideal Writer, respectivamente, nos quais o autor apresenta algumas figuras de linguagem, a fim de realçar o recurso estilístico do texto literário e também a importância do repertório lexical.

Para o autor, a reflexão acerca da linguagem e a força da expressão artística - escrita criativa - poderiam ser estudadas com mais atenção. Assim, ele as incorporou em suas teorias científicas, realçando traços peculiares da estética - simplicidade no estilo e a escrita eficiente -, de modo claro na transmissão de informações.

\subsection{Sobre a tradução}

Considerando o registro linguístico, a escolha lexical e a adequação elocutiva do estilo nos ensaios filosófico-literários de Spencer, a proposta de tradução adotada buscou trabalhar a ideia de equivalência, em língua portuguesa, dessas distintas modalidades discursivas. Para a professora Mona Baker da Universidade de Manchester, Inglaterra,

Para a maioria das línguas, é possível que haja equivalentes dos verbos como falar e dizer; mas para muitos outros verbos podem não ter um equivalente de um tipo mais específico. As línguas, compreensivelmente, tendem a fazer apenas aquelas distinções de significado, os quais são relevantes para seu ambiente particular, seja ele físico, histórico, político, religioso, cultural, econômico, legal, tecnológico, social, seja qualquer outro. $^{2}$

\footnotetext{
${ }^{2}$ Mona Baker, In other words, 1992, p. 18, grifos da autora, tradução minha: "Most languages are likely to have equivalents for the more general verbs of speech such as say and speak, but many
} 
Nesse sentido, e a partir da concepção de equivalência vista pelos Estudos da Tradução ${ }^{3}$, desde a sua perspectiva histórica, Paulo Rónai ressalta que

A arte do tradutor consiste justamente em saber quando pode verter e quando deve procurar equivalências. Mas como não há equivalências absolutas, uma palavra, expressão ou frase do original pode ser frequentemente transportada de duas maneiras, ou mais, sem que possa dizer qual das duas é a melhor. ${ }^{4}$

Assim, no livro selecionado para este trabalho, observou-se a filosofia do autor, especialmente seu pensamento em relação ao uso da linguagem e sua retórica, de modo a oferecer uma proposta de tradução para os ensaios apresentados a seguir.

\section{A tradução}

\subsection{A economia no uso das palavras}

O maior vigor do inglês antigo, ou melhor, do inglês não latino, é o que nos chama a atenção. As diversas razões especiais atribuídas a isso podem ser reduzidas ao motivo geral: a ideia de economia. A mais importante delas é a sua associação precoce. $O$ vocabulário de uma criança é quase inteiramente anglosaxão. Ela diz: eu tenho, não eu possuo, eu gostaria, não eu desejo; ela não reflete, ela pensa; ela não implora por divertimento, mas sim por brincar; ela chama as coisas de boas ou desagradáveis, não de simpáticas ou enfadonhas. Os sinônimos que a criança aprende depois de anos nunca se tornam tão próximos, tão organicamente conectados, às ideias significadas como essas palavras originais usadas na infância; logo, a associação entre elas permanece menos forte. Mas em que uma forte associação entre uma palavra e uma ideia difere de uma fraca? Simplesmente, na maior facilidade e rapidez da ação sugestiva. Não pode ser nada

may not have equivalents for the more specific ones. Languages understandably tend to make only those distinctions in meaning which are relevant to their particular environment, be it physical, historical, political, religious, cultural, economic, legal, technological, social, or otherwise".

${ }^{3}$ Marina N. Menéndez, El concepto de equivalencia, 2012. p. 119.

${ }^{4}$ Paulo Rónai, Escola de tradutores, 1987, p. 23. 
mais que isso. Ambas as palavras, se fossem sinônimas idênticas, possivelmente evocariam a mesma imagem. Afinal, a expressão é ácida deve dar origem ao mesmo pensamento que é azedo; mas, como o termo ácido foi aprendido mais tarde na vida e não foi comumente seguido pelo pensamento simbolizado, logo não desperta de imediato esse pensamento tanto como o termo azedo. Se nos lembrarmos de quão devagar e com que trabalho as ideias favoráveis seguem palavras desconhecidas em outro idioma, do mesmo modo que como a crescente familiaridade com que tais palavras trazem maior rapidez e facilidade de compreensão. Assim, se considerarmos que o mesmo processo deveria ter continuado com as palavras de nossa língua materna desde a infância, nós veríamos claramente que as primeiras locuções aprendidas e mais frequentemente usadas irão, entre outras coisas semelhantes, evocar imagens com menos perda de tempo e energia do que seus últimos sinônimos aprendidos.

A superioridade adicional possuída pelo inglês antigo em sua comparativa brevidade, obviamente, vem sob a mesma generalização. Se for uma vantagem expressar uma ideia no menor número de palavras, então será uma vantagem expressá-la no menor número de sílabas. Se frases tortuosas e palavrões desnecessários distraem a atenção e diminuem a força da impressão produzida, com efeito, as articulações excedentes o fazem. Um certo esforço, embora comumente um pouco digno de nota, deve ser requerido para reconhecer todas as vogais e consoantes. Se, como todos sabem, é cansativo ouvir um palestrante indistinto ou ler um manuscrito mal escrito; e se, como não podemos duvidar, a fadiga é um resultado cumulativo da atenção necessária para capturar sílabas sucessivas; segue-se que a atenção é, nesses casos, absorvida por cada sílaba. E, se isso for verdade, quando as sílabas são difíceis de reconhecimento, também será verdade, embora em menor grau, quando o reconhecimento delas for fácil. Por isso, a falta de palavras saxônicas torna-se uma razão para sua força maior. Uma classificação, no entanto, não deve ser negligenciada. Uma palavra que, por si só, incorpora a parte mais importante da ideia a ser transmitida, principalmente quando essa ideia é emocional, pode, muitas vezes, com vantagem, ser uma 
palavra polissilábica. Assim, parece mais forçado dizer é magnífico do que é grandioso. A palavra vasta não é tão poderosa quanto estupenda. Chamar uma coisa de desagradável não causa o mesmo impacto que chamá-la de nojento.

Todavia, parece haver diversas causas para essa excepcional superioridade de certas palavras longas. Nós podemos atribuí-la, em parte, ao fato de que um epíteto volumoso, que enche a boca e é, por seu tamanho, sugestivo de grandeza ou força, pois testemunha a imensa pomposidade do palavreado sesquipedal. Ou seja, quando um grande poder ou uma intensidade tem de ser sugerido, essa associação de ideias auxilia o efeito. Uma outra causa pode ser que uma palavra de várias sílabas admite uma articulação mais enfática; e como a articulação enfática é um sinal de emoção, a expressividade incomum da coisa nomeada é implicada por ela. Ainda, uma outra causa é que uma palavra longa, da qual as últimas sílabas são geralmente deduzidas assim que a primeira é falada, permite à consciência do ouvinte ter um tempo maior para enfatizar a qualidade predicada; e, como nos casos acima, é para essa qualidade predicada que toda a atenção é voltada; isto é, uma vantagem resulta de mantê-la diante da mente por um tempo apreciável. As razões que nós demos para preferir palavras curtas evidentemente não se sustentam aqui. Nessa conformidade, para tornar nossa generalização bastante correta, devemos dizer que, embora, em certas frases que expressam sentimentos fortes, a palavra que mais especificamente sugere que o sentimento pode, muitas vezes, com vantagem, ser um termo de muitas sílabas ou latino, na imensa maioria dos casos, para cada palavra que serve, mas como um passo para a ideia incorporada por toda a sentença, deve, se possível, ser uma única sílaba ou saxônica.

Mais uma vez, essa causa frequente de força em saxões e outras palavras primitivas - seu caráter imitativo - pode ser similarmente resolvido num motivo mais amplo. Não somente as palavras diretamente imitativas, como respingo, estrondo, zumbido, rugido etc., e aquelas analogicamente imitativas, por exemplo, ásperos, suaves, afiados, contundentes, finos, duros, rochedos etc., têm uma maior ou menor semelhança com as coisas simbolizadas; e, fazendo as 
impressões dos sentidos aliadas às ideias a serem evocadas, elas economizam parte do esforço necessário para invocar tais noções e deixam mais atenção para as próprias ideias.

A economia da energia mental do receptor, na qual são assim resolvíveis as várias causas da força do inglês antigo, pode igualmente ser traçada na superioridade de palavras específicas sobre genéricas. Esses termos concretos produzem impressões mais vívidas do que abstratas, e devem, quando possível, ser usados em vez disso, é uma máxima completa de composição. Como o Dr. Campbell diz: "Quanto mais gerais os termos são, a imagem é mais fraca; quanto mais especiais eles são, logo mais brilhantes". Devemos evitar uma sentença como: Na medida em que as maneiras, costumes e diversões de uma nação são cruéis e bárbaros, os regulamentos de seu código penal serão severos. E no lugar disso deveríamos escrever: Na medida em que os homens se deleitam em batalhas, lutas de touro e combates de gladiadores, eles irão punir pendurando, queimando e a cavalete.

Essa superioridade de expressões específicas deve-se claramente à economia do esforço necessário para traduzir palavras em pensamentos. Como não pensamos em gerais, mas em particulares, como exemplo, sempre que qualquer classe de coisas é referida, nós a representamos a nós mesmos, chamando à mente os membros individuais dela; segue-se que, nesse sentido, quando uma palavra abstrata é usada, o portador ou leitor tem que escolher, a partir de seu estoque de imagens, uma ou mais, pelas quais ele pode imaginar a si mesmo o gênero mencionado. Ao fazer isso, algum atraso deve surgir a fim de que a força seja gasta; e se, empregando um termo específico, uma imagem apropriada pode ser sugerida, uma economia é alcançada e uma impressão mais vívida é produzida. 


\subsection{O efeito da poesia explicado}

Antes de indagar se a lei do efeito, até o momento delineada, explica a superioridade da poesia à prosa, será necessário notar algumas causas suplementares da força na expressão, as quais ainda não foram mencionadas. Essas não são, propriamente falando, causas adicionais; mas as secundárias, provenientes daqueles resultados e seus reflexos já especificados. Inicialmente, nós podemos observar que a excitação mental espontaneamente estimula o uso dessas formas de discurso, que foram apontadas como as mais eficazes. Caía fora daquil ou fique longe dele! são enunciados naturais de cidadãos irritados em uma reunião conturbada. Outrossim, um navegador, descrevendo uma terrível tempestade que ele testemunhara, chegaria a um clímax como: Estouraram as cordas e o mastro veio abaixo. A frase que expressa surpresa pode ser ouvida como: Nunca houve algo parecido!, e todas essas proposições são construídas, como observaremos, conforme a lógica direta. A propósito, todos nós sabemos que figuras de linguagem são atribuídas às pessoas animadas. Em verdade, o vitupério do indigno abunda com elas e, muitas vezes, isso consiste em um pouco mais. Cruel, rude, ladino, vilão degolado, esses e outros nomes usados como metáforas e epítetos metafóricos, imediatamente lembram-nos as brigas de rua. Pode-se notar inclusive que a extrema brevidade é outra característica da linguagem apaixonada. Comumente, as sentenças são incompletas; as partículas são omitidas, e as palavras importantes sempre são deixadas para serem coletadas a partir dum contexto. A grande admiração não se exprime numa proposição precisa, como É bela; mas na simples exclamação: Linda!. Aquele que, ao ler uma missiva de um advogado, dissesse: Vil tratante!, pensaria com raiva, enquanto que, se dissesse Ele é um vil tratante!, implicaria frieza comparativa. Deste modo, nós vemos o equivalente acontecer com a ordem das palavras, no uso frequente de figuras de linguagem e na extrema concisão, cujas expressões naturais de excitação seguem conforme as condições teóricas de uma expressão convincente. 
Por conseguinte, as formas acuradas de discurso adquirem uma força suplementar pelo mecanismo de associação. Sendo habitualmente ouvidas em conexão com impressões mentais vivas, e sendo regularmente encontradas nos escritos mais influentes, essas associações passam a ter em si uma espécie de força. As emoções que foram de tempo em tempo produzidas por pensamentos expressivos envolvidos nessas formas são parcialmente despertadas pelas próprias formas. Elas criam um certo grau de exaltação; elas induzem uma simpatia preparatória; e quando as ideias significativas que se busca são alcançadas, elas são compreendidas de forma mais vívida.

O uso contínuo dessas formas de expressão, as quais são igualmente fortes por si sós e o tanto quanto a partir de suas associações, produz a composição particularmente considerável que chamamos de poesia. A poesia, como notaremos, geralmente adota esses símbolos do pensamento e os métodos de usálos que o instinto e a análise concordam em escolhê-los como mais eficazes, e torna-se poesia em virtude de fazê-lo. Ao retomar aos vários exemplos citados, ver-se-á que a forma direta ou invertida da oração predomina neles, em um nível quase que inadmissível na prosa. $E$ isso ocorre não apenas na frequência, mas no que é chamado de violência das inversões, na qual a distinção será notada. Nessa acepção, nós podemos reconhecer a mesma verdade no uso abundante de figuras de linguagem. Metáforas, símiles, hipérboles e personificações são as cores dos poetas, as quais eles têm a liberdade para empregá-las quase sem limites. Caracterizamos como poética a prosa que usa essas figuras de estilo com alguma constância, e a condenamos como excessivamente florida ou afetada muito antes que elas ocorrem com a profusão permitida no poema. Além disso, observa-se que na brevidade - outro requisito de forte expressão que a teoria aponta e a emoção realiza espontaneamente - a fraseologia poética difere da fraseologia ordinária. Períodos imperfeitos são contínuos; as elisões são permanentes; e muitas das palavras menores, que seriam consideradas essenciais na prosa, são dispensadas.

Nesse sentido, a poesia, considerada como um instrumento de reflexão, é especialmente admirável em parte porque ela obedece a todas as leis do discurso 
efetivo, também em parte porque, ao fazê-lo, imita as expressões naturais da emoção. Enquanto a questão corporificada é emoção idealizada, o instrumento é a linguagem idealizada da emoção. À medida que o compositor musical capta as cadências nas quais nossos sentimentos de alegria e simpatia, tristeza e desespero, se liberam; e desses inícios florescem melodias, sugerindo fases mais elevadas desses sentimentos; então o poeta desenvolve-se a partir das expressões típicas em que os homens expressam paixão e sentimento sob formas escolhidas de combinação verbal, em que a paixão concentrada e o sentimento podem ser adequadamente apresentados.

Há uma peculiaridade da poesia que conduz muito ao seu efeito - a peculiaridade que é, de fato, comumente pensada como uma característica própria - ainda a ser considerada: a sua estrutura rítmica. Isso, embora pareça improvável, será encontrado sob a mesma generalização a respeito das outras. Como cada uma dessas estruturas rítmicas é uma idealização da linguagem natural de forte emoção, que é conhecida por ser mais ou menos métrica se a emoção não for violenta demais, logo cada uma delas é como uma economia da atenção do leitor ou do ouvinte. Segundo o tom e os modos peculiares, os quais adotamos ao pronunciar a linguagem versificada, podem-se discernir sua relação com os sentimentos; e o prazer que o seu movimento medido nos dá é atribuível à facilidade comparativa com a qual as palavras metricamente arranjadas podem ser identificadas.

Essa última exposição dificilmente será imediatamente admitida, no entanto, uma pequena explicação mostrará sua razoabilidade. E se, como nós vimos, há um gasto de energia mental no mero ato de ouvir as articulações verbais ou nessa repetição silenciosa que nela ocorre a leitura - supondo que as capacidades perceptivas devem estar em exercício ativo para identificar cada sílaba -, então, qualquer modo de combinar palavras que apresente uma repetição regular de certos traços que a mente pode antecipar diminuirá essa preocupação sobre a atenção requerida pela total irregularidade da prosa. Assim como o corpo, ao receber uma série de concussões variáveis, deve-se manter os 
músculos prontos para enfrentar as mais violentas, o que é como não saber quando isso pode acontecer, ao passo que a mente, ao receber articulações desiguais, deve manter seus perceptivos suficientemente ativos para reconhecer os sons captados com menos facilidade. E, conforme as comoções se repetem em uma ordem definida, o corpo pode administrar suas forças ajustando a resistência necessária para cada concussão. Por consequência, se as sílabas estiverem harmonizadas, ritmicamente, a mente pode economizar suas energias antecipando a atenção necessária para cada sílaba.

Apesar de essa ideia ser talvez exagerada, um pouco de introspecção irá aprová-la. O fato de nós aproveitarmos a linguagem métrica para ajustar nossas capacidades perceptivas à força das articulações esperadas fica claro pelo fato de que estamos impedidos de interromper a versificação. Isso que, tanto quanto no fundo de um lance de escadas, um passo mais ou menos do que contamos nos dá um choque. Assim o mesmo acontece com um acento gráfico errado ou uma sílaba supranumerária. No primeiro caso, nós sabemos que há um pré-ajuste equivocado e dificilmente podemos duvidar que haja um no outro; mas, se constantemente adaptarmos nossas percepções ao movimento medido do verso, a analogia física mencionada acima torna provável que, ao fazê-lo, nós economizemos atenção; e, portanto, essa linguagem métrica é mais eficaz que a prosa, porque nos permite pensá-la desta forma.

Se houvesse espaço, valeria a pena perguntar se o prazer que nós damos à rima, igualmente ao que damos à eufonia, não são, parcialmente, atribuíveis à mesma causa geral.

\subsection{Explicação do clímax, antítese e anticlímax}

Tendo em mente essas verdades gerais, nós devemos estar em condições de entender certas causas de efeito na composição, as quais precisam ser consideradas. Toda percepção recebida e toda concepção percebida, acarretará-lo 
uma certa quantidade de desperdício, ou, como diria Liebig, alguma mudança de matéria no cérebro; e, a eficiência das faculdades sujeitas a esse desperdício é, assim, temporariamente, embora muitas vezes momentaneamente, diminuída, a incapacidade parcial resultante deve afetar os atos de percepção e concepção que imediatamente os sucedem. Portanto, podemos esperar que a vivacidade com as quais as imagens são realizadas irá, em muitos casos, depender da ordem de sua apresentação, mesmo quando uma ordem é tão conveniente para o entendimento quanto a outra.

Há diversos fatos que ilustram e explicam isso, e o clímax é um deles. $\mathrm{O}$ efeito marcante obtido, colocando a mais notável de todas as séries de imagens, e a fraqueza - muitas vezes a fraqueza ridícula -, produzida pela reversão desse arranjo, dependem da lei geral indicada. Do mesmo modo que, imediatamente depois de olharmos para o sol, não podemos perceber a luz do fogo, enquanto, olhando primeiro para o fogo e depois para o sol, podemos perceber ambos; assim, depois de receber um pensamento brilhante, pesado ou terrível, não podemos apreciar um menos brilhante, menos pesado ou menos terrível, enquanto, ao inverter a ordem, podemos apreciar cada um deles isoladamente. Em antítese, novamente, podemos reconhecer a mesma verdade geral. A oposição de dois pensamentos que são o inverso um do outro em algum traço proeminente assegura um efeito impressionante; e faz isso dando um relaxamento momentâneo às capacidades abordadas. Se, após uma série de imagens de um personagem comum, apelando em grau moderado ao sentimento de reverência, aprovação ou beleza, a mente lhe apresentou uma imagem muito insignificante, muito indigna ou muito feia; a propriedade de reverência, aprovação ou beleza, conforme for o caso, não tendo nada para fazer, tende a retomar seu pleno poder; e imediatamente apreciará uma imagem vasta, admirável ou bonita melhor do que seria de qualquer outra forma. Sob outra perspectiva, cuja ideia de absurdo devido à extrema insignificância é para ser produzida, talvez intensifique intensamente colocando-a depois de algo altamente impressionante, especialmente se a forma da frase implica que algo ainda mais impressionante 
está chegando. Uma boa ilustração do efeito obtido por apresentar uma ideia mesquinha a uma consciência que ainda não se recuperou do choque de uma ideia excitante ocorre em um esboço de Honoré de Balzac. Seu herói escreve a uma amante, que fora fria com ele, a seguinte carta:

Madame, Votre conduite m'étonne autant qu'elle m'afflige. Non contente de me déchirer le coeur par vos dédains vous avez l'indélicatesse de me retenir une brosse à dents, que mes moyens ne me permettent pas de remplacer, mes propriétés etant grevées d'hypothèques.

Adieu, trop, belle et trop ingrate ainie! Puissions nous nous revoir dans un monde meilleur!

\section{Charles Edouard.}

Assim, vemos que os fenômenos do clímax, antítese e anticlímax, igualmente, resultam desse princípio geral. Por mais improváveis que possam parecer essas variações momentâneas de suscetibilidade, não podemos duvidar de sua ocorrência quando contemplamos as variações análogas na suscetibilidade dos sentidos. Referindo-se, mais uma vez, a fenômenos de visão, todos sabem que uma parte preta num fundo branco parece mais escura e uma parte branca num chão preto parece mais branca do que em qualquer outro lugar. Como a negritude e a brancura devem ser realmente as mesmas, a única causa determinável para isso é uma diferença em suas ações sobre nós, dependente dos diferentes estados de nossas capacidades. É simplesmente uma antítese visual.

\subsection{A necessidade de variedade}

No entanto, essa extensão do princípio geral da economia - essa condição adicional para a composição efetiva, de que a sensibilidade das capacidades deve ser continuamente administrada - inclui muito mais do que já foi sugerido. Isso 
implica não apenas em certos arranjos e certas justaposições de ideias conectadas são melhores; mas que alguns modos de dividir e apresentar um assunto serão mais notáveis do que outros; e isso também independentemente de sua coesão lógica. Isso mostra, contudo, porque devemos progredir do menos interessante para o mais interessante; e porque não apenas a composição como um todo, pois cada uma de suas porções sucessivas deve tender para um clímax. Ao mesmo tempo, proíbe-se a continuidade prolongada do mesmo tipo de pensamento ou a produção repetida de efeitos semelhantes. Ele nos adverte contra o erro cometido tanto por Alexander Pope em seus poemas quanto por Francis Bacon em seus ensaios. O erro, a saber, de empregar constantemente formas de expressão forçadas, e aponta que a postura mais fácil torna-se fatigante, pois é com prazer trocado por um menos fácil, então, as sentenças mais perfeitamente construídas logo se cansarão, e o alívio será dado usando aquelas de um tipo inferior.

Além disso, nós podemos inferir daí não somente que devemos evitar geralmente combinar nossas palavras de uma única maneira, por melhor que seja, ou elaborar nossas figuras e ilustrações de um único jeito, por mais revelador que seja; mas sim devemos evitar algo como aderência uniforme, mesmo às condições mais amplas de efeito. Não devemos fazer com que cada seção do nosso assunto progrida no interesse; nem sempre devemos nos elevar ao clímax. Como vimos, em sentenças únicas, raramente é permitido preencher todas as condições para se fortalecer. Assim, nas seções maiores de uma composição, não devemos nos conformar inteiramente à lei indicada. Devemos subordinar o efeito do componente ao efeito total.

Ao decidir como é prático executar os princípios da composição artística, podemos obter ajuda tendo em mente um fato já mencionado, a adequação de certos arranjos verbais para certos tipos de pensamento. Essa variedade constante no modo de apresentar ideias que a teoria exige resultará, em grande parte, de uma adaptação habilidosa da forma à matéria. Nesse sentido, nós vimos como a sentença direta ou invertida é usada espontaneamente por pessoas entusiasmadas; e como sua linguagem também é caracterizada por figuras de 
estilo e por extrema brevidade. Por isso, estas podem com vantagem predominar em passagens emocionais e podem aumentar à medida que a emoção também aumenta. Por outro lado, para ideias complexas, por exemplo, a sentença indireta parece o melhor veículo. Na conversa, a excitação produzida pela abordagem aproximada de uma conclusão desejada, muitas vezes se mostrará em uma série de frases curtas e precisas, enquanto que, ao imprimir uma visão já enunciada, geralmente tornamos nossos períodos volumosos empilhando pensamento sobre pensamento. Esses modos naturais de procedimento podem servir como guias para a escrita. A observação aguçada e a análise hábil, semelhantemente, detectariam outras peculiaridades de expressão produzidas por outras atitudes mentais; e, prestando a devida atenção a todos esses traços, um escritor dotado de versatilidade suficiente poderia fazer alguma abordagem a um trabalho completamente organizado.

\subsection{O escritor ideal}

Este tipo de composição da qual a lei do efeito aponta como a perfeita é aquela que o alto gênio tende naturalmente a produzir. Quando descobrimos que os tipos de sentenças, teoricamente, melhores são aquelas geralmente empregadas por mentes superiores e por mentes inferiores quando a excitação as eleva; então descobriremos que a forma ideal para um poema, ensaio ou ficção é aquela que o escritor ideal evoluiria espontaneamente. Naquela em quem os poderes de expressão responderiam plenamente ao estado de sentimento, inconscientemente, usaria essa variedade no modo de apresentar seus pensamentos, como a Arte exige. Esse emprego constante de uma espécie de fraseologia, a qual todos têm agora de lutar contra, implica uma faculdade de linguagem não desenvolvida. Ter um estilo específico é ser pobre na fala. Se nos lembrarmos que, no passado distante, os homens tinham apenas substantivos e verbos para transmitir suas ideias, e que, desde então, o crescimento foi para um número maior de implementos de pensamento e, consequentemente, em direção 
a uma maior complexidade e variedade em suas combinações; podemos inferir que estamos agora, em nosso uso de sentenças, muito mais do que o homem primitivo usava em palavras; e que a continuação do processo que até agora ocorreu deve produzir uma crescente heterogeneidade em nossos modos de expressão. Atualmente, em boa natureza, o jogo das feições, os tons da voz e suas cadências variam em harmonia com todo pensamento proferido; assim, em alguém possuidor de um poder de fala totalmente desenvolvido, o molde no qual cada combinação de palavras é lançada variará da mesma forma e será apropriado ao sentimento.

Um homem perfeitamente dotado deve inconscientemente escrever em todos os estilos; nós podemos deduzir dessa consideração que os estilos se originam. Por que Johnson é pomposo e Goldsmith é simples? Por que um autor é abrupto, outro rítmico, outro conciso? Evidentemente, em cada caso o modo habitual de expressão deve depender do equilíbrio habitual da natureza. Os sentimentos predominantes têm por uso treinado o intelecto para representá-los. Mas, embora por muito tempo, embora inconsciente, a disciplina tenha feito isso eficientemente, resta da falta de prática, incapaz de fazer o mesmo com os sentimentos menos ativos; e quando estes estão excitados, as formas verbais habituais passam por pequenas modificações. Que os poderes da fala sejam plenamente desenvolvidos, no entanto, que a capacidade do intelecto de expressar as emoções seja completa; e essa fixidez de estilo desaparecerá. 0 escritor perfeito se expressará como Junius, quando estiver na mente de Junius; quando ele se sentir como Lamb sentiu, usará um discurso familiar; e vai cair na robustez de Carlyle quando este estiver em um clima carlyleano. Ora ele será rítmico, ora irregular e, assim, a sua linguagem será clara e ornamentada. Às vezes, suas sentenças serão equilibradas e, outras vezes, assimétricas, por um tempo haverá uma uniformidade considerável, e, com efeito, uma grande variedade. Seu modo de expressão, naturalmente, respondendo ao seu estado de sentimento, fluirá de sua caneta uma composição mudando no mesmo grau em que os aspectos de seu assunto mudam. Ele irá, portanto, sem esforço, conforme 
o que temos visto como as leis de efeito. E, enquanto seu trabalho apresenta ao leitor essa variedade necessária para evitar o esforço contínuo das mesmas capacidades, ele também responderá à descrição de todos os produtos altamente organizados, tanto do homem como da natureza; não será uma série de peças semelhantes simplesmente colocando-se em justaposição, mas um todo composto de partes diferentes que são mutuamente dependentes.

\section{Ensaios originais}

\subsection{Economy in the Use of Words}

$\S 5$. The greater forcibleness of Saxon English, or rather non-Latin English, first claims our attention. The several special reasons assignable for this may all be reduced to the general reason-economy. The most important of them is early association. A child's vocabulary is almost wholly Saxon. He says, I have, not I possess--I wish, not I desire; he does not reflect, he thinks; he does not beg for amusement, but for play; he calls things nice or nasty, not pleasant or disagreeable. The synonyms which he learns in after years, never become so closely, so organically connected with the ideas signified, as do these original words used in childhood; and hence the association remains less strong. But in what does a strong association between a word and an idea differ from a weak one? Simply in the greater ease and rapidity of the suggestive action. It can be in nothing else. Both of two words, if they be strictly synonymous, eventually call up the same image. The expression-It is acid, must in the end give rise to the same thought as-It is sour; but because the term acid was learnt later in life, and has not been so often followed by the thought symbolized, it does not so readily arouse that thought as the term sour. If we remember how slowly and with what labour the appropriate ideas follow unfamiliar words in another language, and how increasing familiarity with such words brings greater rapidity and ease of comprehension; and if we consider that the same process must have gone on with the words of our mother tongue from childhood upwards, we shall clearly 
see that the earliest learnt and oftenest used words, will, other things equal, call up images with less loss of time and energy than their later learnt synonyms.

§6. The further superiority possessed by Saxon English in its comparative brevity, obviously comes under the same generalization. If it be an advantage to express an idea in the smallest number of words, then will it be an advantage to express it in the smallest number of syllables. If circuitous phrases and needless expletives distract the attention and diminish the strength of the impression produced, then do surplus articulations do so. A certain effort, though commonly an inappreciable one, must be required to recognize every vowel and consonant. If, as all know, it is tiresome to listen to an indistinct speaker, or read a badlywritten manuscript; and if, as we cannot doubt, the fatigue is a cumulative result of the attention needed to catch successive syllables; it follows that attention is in such cases absorbed by each syllable. And if this be true when the syllables are difficult of recognition, it will also be true, though in a less degree, when the recognition of them is easy. Hence, the shortness of Saxon words becomes a reason for their greater force. One qualification, however, must not be overlooked. A word which in itself embodies the most important part of the idea to be conveyed, especially when that idea is an emotional one, may often with advantage be a polysyllabic word. Thus it seems more forcible to say, "It is magnificent," than "It is grand." The word vast is not so powerful a one as stupendous. Calling a thing nasty is not so effective as calling it disgusting.

$\S 7$. There seem to be several causes for this exceptional superiority of certain long words. We may ascribe it partly to the fact that a voluminous, mouth-filling epithet is, by its very size, suggestive of largeness or strength; witness the immense pomposity of sesquipedalian verbiage: and when great power or intensity has to be suggested, this association of ideas aids the effect. A further cause may be that a word of several syllables admits of more emphatic articulation; and as emphatic articulation is a sign of emotion, the unusual impressiveness of the thing named is implied by it. Yet another cause is that a long word (of which the latter syllables are generally inferred as soon as the first 
are spoken) allows the hearer's consciousness a longer time to dwell upon the quality predicated; and where, as in the above cases, it is to this predicated quality that the entire attention is called, an advantage results from keeping it before the mind for an appreciable time. The reasons which we have given for preferring short words evidently do not hold here. So that to make our generalization quite correct we must say, that while in certain sentences expressing strong feeling, the word which more especially implies that feeling may often with advantage be a many-syllabled or Latin one; in the immense majority of cases, each word serving but as a step to the idea embodied by the whole sentence, should, if possible, be a one-syllabled or Saxon one.

$\S$ 8. Once more, that frequent cause of strength in Saxon and other primitive words-their imitative character may be similarly resolved into the more general cause. Both those directly imitative, as splash, bang, whiz, roar, \&c., and those analogically imitative, as rough, smooth, keen, blunt, thin, hard, crag, \&c., have a greater or less likeness to the things symbolized; and by making on the senses impressions allied to the ideas to be called up, they save part of the effort needed to call up such ideas, and leave more attention for the ideas themselves.

$\S 9$. The economy of the recipient's mental energy, into which are thus resolvable the several causes of the strength of Saxon English, may equally be traced in the superiority of specific over generic words. That concrete terms produce more vivid impressions than abstract ones, and should, when possible, be used instead, is a thorough maxim of composition. As Dr. Campbell says, "The more general the terms are, the picture is the fainter; the more special they are, 'tis the brighter." We should avoid such a sentence as:-"In proportion as the manners, customs, and amusements of a nation are cruel and barbarous, the regulations of their penal code will be severe." And in place of it we should write:-"In proportion as men delight in battles, bull-fights, and combats of gladiators, will they punish by hanging, burning, and the rack." 
$\S 10$. This superiority of specific expressions is clearly due to a saving of the effort required to translate words into thoughts. As we do not think in generals but in particulars-as, whenever any class of things is referred to, we represent it to ourselves by calling to mind individual members of it; it follows that when an abstract word is used, the bearer or reader has to choose from his stock of images, one or more, by which he may figure to himself the genus mentioned. In doing this, some delay must arise some force be expended; and if, by employing a specific term, na appropriate image can be at once suggested, an economy is achieved, and a more vivid impression produced.

\subsection{The Effect of Poetry Explained}

$\S 50$. Before inquiring whether the law of effect, thus far traced, explains the superiority of poetry to prose, it will be needful to notice some supplementary causes of force in expression, that have not yet been mentioned. These are not, properly speaking, additional causes; but rather secondary ones, originating from those already specified reflex results of them. In the first place, then, we may remark that mental excitement spontaneously prompts the use of those forms of speech which have been pointed out as the most effective. "Out with him!" "Away with him!" are the natural utterances of angry citizens at a disturbed meeting. A voyager, describing a terrible storm he had witnessed, would rise to some such climax as-"Crack went the ropes and down came the mast." Astonishment may be heard expressed in the phrase-"Never was there such a sight!" All of which sentences are, it will be observed, constructed after the direct type. Again, every one knows that excited persons are given to figures of speech. The vituperation of the vulgar abounds with them: often, indeed, consists of little else. "Beast," "brute," "gallows rogue," "cut-throat villain," these, and other like metaphors and metaphorical epithets, at once call to mind a street quarrel. Further, it may be noticed that extreme brevity is another characteristic of passionate language. The sentences are generally incomplete; the particles are 
omitted; and frequently important words are left to be gathered from the context. Great admiration does not vent itself in a precise proposition, as-"It is beautiful"; but in the simple exclamation-"Beautiful!" He who, when reading a lawyer's letter, should say, "Vile rascal!" would be thought angry; while, "He is a vile rascal!" would imply comparative coolness. Thus we see that alike in the order of the words, in the frequent use of figures, and in extreme conciseness, the natural utterances of excitement conform to the theoretical conditions of forcible expression.

$\S 51$. Hence, then, the higher forms of speech acquire a secondary strength from association. Having, in actual life, habitually heard them in connection with vivid mental impressions, and having been accustomed to meet with them in the most powerful writing, they come to have in themselves a species of force. The emotions that have from time to time been produced by the strong thoughts wrapped up in these forms, are partially aroused by the forms themselves. They create a certain degree of animation; they induce a preparatory sympathy, and when the striking ideas looked for are reached, they are the more vividly realized

$\S 52$. The continuous use of these modes of expression that are alike forcible in themselves and forcible from their associations, produces the peculiarly impressive species of composition which we call poetry. Poetry, we shall find, habitually adopts those symbols of thought, and those methods of using them, which instinct and analysis agree in choosing as most effective, and becomes poetry by virtue of doing this. On turning back to the various specimens that have been quoted, it will be seen that the direct or inverted form of sentence predominates in them; and that to a degree quite inadmissible in prose. And not only in the frequency, but in what is termed the violence of the inversions, will this distinction be remarked. In the abundant use of figures, again, we may recognize the same truth. Metaphors, similes, hyperboles, and personifications, are the poet's colours, which he has liberty to employ almost without limit. We characterize as "poetical" the prose which uses these 
appliances of language with any frequency, and condemn it as "over florid" or "affected" long before they occur with the profusion allowed in verse. Further, let it be remarked that in brevity-the other requisite of forcible expression which theory points out, and emotion spontaneously fulfils-poetical phraseology similarly differs from ordinary phraseology. Imperfect periods are frequent; elisions are perpetual; and many of the minor words, which would be deemed essential in prose, are dispensed with.

$\S 53$. Thus poetry, regarded as a vehicle of thought, is especially impressive partly because it obeys all the laws of effective speech, and partly because in so doing it imitates the natural utterances of excitement. While the matter embodied is idealized emotion, the vehicle is the idealized language of emotion. As the musical composer catches the cadences in which our feelings of joy and sympathy, grief and despair, vent themselves, and out of these germs evolves melodies suggesting higher phases of these feelings; I so, the poet develops from the typical expressions in which men utter passion and sentiment, those choice forms of verbal combination in which concentrated passion and sentiment may be fitly presented.

$\S 54$. There is one peculiarity of poetry conducing much to its effect-the peculiarity which is indeed usually thought its characteristic one-still remaining to be considered: we mean its rhythmical structure. This, improbable though it seems, will be found to come under the same generalization with the others. Like each of them, it is an idealization of the natural language of strong emotion, which is known to be more or less metrical if the emotion be not too violent; and like each of them it is an economy of the reader's or hearer's attention. In the peculiar tone and manner we adopt in uttering versified language, may be discerned its relationship to the feelings; and the pleasure which its measured movement gives us, is ascribable to the comparative ease with which words metrically arranged can be recognized. 
$\S 55$. This last position will scarcely be at once admitted; but a little explanation will show its reasonableness. For if, as we have seen, there is an expenditure of mental energy in the mere act of listening to verbal articulations, or in that silent repetition of them which goes on in reading-if the perceptive faculties must be in active exercise to identify every syllable-then, any mode of so combining words as to present a regular recurrence of certain traits which the mind can anticipate, will diminish that strain upon the attention required by the total irregularity of prose. Just as the body, in receiving a series of varying concussions, must keep the muscles ready to meet the most violent of them, as not knowing when such may come; so, the mind in receiving unarranged articulations, must keep its perceptives active enough to recognize the least easily caught sounds. And as, if the concussions recur in a definite order, the body may husband its forces by adjusting the resistance needful for each concussion; so, if the syllables be rhythmically arranged, the mind may economize its energies by anticipating the attention required for each syllable.

$\S 56$. Far-fetched though this idea will perhaps be thought, a little introspection will countenance it. That we do take advantage of metrical language to adjust our perceptive faculties to the force of the expected articulations, is clear from the fact that we are balked by halting versification. Much as at the bottom of a flight of stairs, a step more or less than we counted upon gives us a shock; so, too, does a misplaced accent or a supernumerary syllable. In the one case, we know that there is an erroneous preadjustment; and we can scarcely doubt that there is one in the other. But if we habitually preadjust our perceptions to the measured movement of verse, the physical analogy above given renders it probable that by so doing we economize attention; and hence that metrical language is more effective than prose, because it enables us to do this.

$\S 57$. Were there space, it might be worthwhile to inquire whether the pleasure we take in rhyme, and also that which we take in euphony, axe not partly ascribable to the same general cause. 


\subsection{Explanation of Climax, Antithesis, and Anticlimax}

$\S 61$. Keeping in mind these general truths, we shall be in a condition to understand certain causes of effect in composition now to be considered. Every perception received, and every conception realized, entailing some amount of waste-or, as Liebig would say, some change of matter in the brain; and the efficiency of the faculties subject to this waste being thereby temporarily, though often but momentarily, diminished; the resulting partial inability must affect the acts of perception and conception that immediately succeed. And hence we may expect that the vividness with which images are realized will, in many cases, depend on the order of their presentation: even when one order is as convenient to the understanding as the other.

$\S 62$. There are sundry facts which alike illustrate this, and are explained by it. Climax is one of them. The marked effect obtained by placing last the most striking of any series of images, and the weakness-often the ludicrous weakness-produced by reversing this arrangement, depends on the general law indicated. As immediately after looking at the sun we cannot perceive the light of a fire, while by looking at the fire first and the sun afterwards we can perceive both; so, after receiving a brilliant, or weighty, or terrible thought, we cannot appreciate a less brilliant, less weighty, or less terrible one, while, by reversing the order, we can appreciate each. In Antithesis, again, we may recognize the same general truth. The opposition of two thoughts that are the reverse of each other in some prominent trait, insures an impressive effect; and does this by giving a momentary relaxation to the faculties addressed. If, after a series of images of an ordinary character, appealing in a moderate degree to the sentiment of reverence, or approbation, or beauty, the mind has presented to it a very insignificant, a very unworthy, or a very ugly image; the faculty of reverence, or approbation, or beauty, as the case may be, having for the time nothing to do, tends to resume its full power; and will immediately afterwards appreciate a vast, admirable, or beautiful image better than it would otherwise do. Conversely, where the idea of absurdity due to extreme insignificance is to 
be produced, it maybe greatly intensified by placing it after something highly impressive: especially if the form of phrase implies that something still more impressive is coming. A good illustration of the effect gained by thus presenting a petty idea to a consciousness that has not yet recovered from the shock of an exciting one, occurs in a sketch by Balzac. His hero writes to a mistress who has cooled towards him the following letter:

"Madame, Votre conduite m'étonne autant qu'elle m'afflige Non contente de me déchirer le coeur par vos dédains vous avez l'indélicatesse de me retenir une brosse à dents, que mes moyens ne me permettent pas de remplacer, mes propriétés etant grevées d'hypothèques

"Adieu, trop, belle et trop ingrate ainie! Puissions nous nous revoir dans un monde meilleur!

\section{"Charles Edouard"}

$\S$ 63. Thus we see that the phenomena of Climax, Antithesis, and Anticlimax, alike result from this general principle. Improbable as these momentary variations in susceptibility may seem, we cannot doubt their occurrence when we contemplate the analogous variations in the susceptibility of the senses. Referring once more to phenomena of vision, every one knows that a patch of black on a white ground looks blacker, and a patch of white on a black ground looks whiter, than elsewhere. As the blackness and the whiteness must really be the same, the only assignable cause for this is a difference in their actions upon us, dependent upon the different states of our faculties. It is simply a visual antithesis.

\subsection{Need of Variety}

$\S 64$. But this extension of the general principle of economy-this further condition to effective composition, that the sensitiveness of the faculties must be 
continuously husbanded-includes much more than has been yet hinted. It implies not only that certain arrangements and certain juxtapositions of connected ideas are best; but that some modes of dividing and presenting a subject will be more striking than others; and that, too, irrespective of its logical cohesion. It shows why we must progress from the less interesting to the more interesting; and why not only the composition as a whole, but each of its successive portions, should tend towards a climax. At the same time, it forbids long continuity of the same kind of thought, or repeated production of like effects. It warns us against the error committed both by Pope in his poems and by Bacon in his essays-the error, namely, of constantly employing forcible forms of expression: and it points out that as the easiest posture by and by becomes fatiguing,

and is with pleasure exchanged for one less easy, so, the most perfectlyconstructed sentences will soon weary, and relief will be given by using those of an inferior kind.

$\S 65$. Further, we may infer from it not only that we should avoid generally combining our words in one manner, however good, or working out our figures and illustrations in one way, however telling; but that we should avoid anything like uniform adherence, even to the wider conditions of effect. We should not make every section of our subject progress in interest; we should not always rise to a climax. As we saw that, in single sentences, it is but rarely allowable to fulfill all the conditions to strength; so, in the larger sections of a composition we must not often conform entirely to the law indicated. We must subordinate the component effect to the total effect.

$\S 66$. In deciding how practically to carry out the principles of artistic composition, we may derive help by bearing in mind a fact already pointed outthe fitness of certain verbal arrangements for certain kinds of thought. That constant variety in the mode of presenting ideas which the theory demands, will in a great degree result from a skilful adaptation of the form to the matter. We 
saw how the direct or inverted sentence is spontaneously used by excited people; and how their language is also characterized by figures of speech and by extreme brevity. Hence these may with advantage predominate in emotional passages; and may increase as the emotion rises. On the other hand, for complex ideas, the indirect sentence seems the best vehicle. In conversation, the excitement produced by the near approach to a desired conclusion, will often show itself in a series of short, sharp sentences; while, in impressing a view already enunciated, we generally make our periods voluminous by piling thought upon thought. These natural modes of procedure may serve as guides in writing. Keen observation and skilful analysis would, in like manner, detect further peculiarities of expression produced by other attitudes of mind; and by paying due attention to all such traits, a writer possessed of sufficient versatility might make some approach to a completely-organized work.

\subsection{The Ideal Writer}

$\S 67$. This species of composition which the law of effect points out as the perfect one, is the one which high genius tends naturally to produce. As we found that the kinds of sentences which are theoretically best, are those generally employed by superior minds, and by inferior minds when excitement has raised them; so, we shall find that the ideal form for a poem, essay, or fiction, is that which the ideal writer would evolve spontaneously. One in whom the powers of expression fully responded to the state of feeling, would unconsciously use that variety in the mode of presenting his thoughts, which Art demands. This constant employment of one species of phraseology, which all have now to strive against, implies an undeveloped faculty of language. To have a specific style is to be poor in speech. If we remember that, in the far past, men had only nouns and verbs to convey their ideas with, and that from then to now the growth has been towards a greater number of implements of thought, and consequently towards a greater complexity and variety in their 
combinations; we may infer that we are now, in our use of sentences, much what the primitive man was in his use of words; and that a continuance of the process that has hitherto gone on, must produce increasing heterogeneity in our modes of expression. As now, in a fine nature, the play of the features, the tones of the voice and its cadences, vary in harmony with every thought uttered; so, in one possessed of a fully developed power of speech, the mould in which each combination of words is cast will similarly vary with, and be appropriate to the sentiment.

$\S 68$. That a perfectly endowed man must unconsciously write in all styles, we may infer from considering how styles originate. Why is Johnson pompous, Goldsmith simple? Why is one author abrupt, another rhythmical, another concise? Evidently in each case the habitual mode of utterance must depend upon the habitual balance of the nature. The predominant feelings have by use trained the intellect to represent them. But while long, though unconscious, discipline has made it do this efficiently, it remains from lack of practice, incapable of doing the same for the less active feelings; and when these are excited, the usual verbal forms undergo but slight modifications. Let the powers of speech be fully developed, however-let the ability of the intellect to utter the emotions be complete; and this fixity of style will disappear. The perfect writer will express himself as Junius, when in the Junius frame of mind; when he feels as Lamb felt, will use a like familiar speech; and will fall into the ruggedness of Carlyle when in a Carlylean mood. Now he will be rhythmical and now irregular; here his language will be plain and there ornate; sometimes his sentences will be balanced and at other times unsymmetrical; for a while there will be considerable sameness, and then again great variety. His mode of expression naturally responding to his state of feeling, there will flow from his pen a composition changing to the same degree that the aspects of his subject change. He will thus without effort conform to what we have seen to be the laws of effect. And while his work presents to the reader that variety needful to prevent continuous exertion of the same faculties, it will also answer to the 
description of all highly organized products, both of man and of nature: it will be not a series of like parts simply placed in juxtaposition, but one whole made up of unlike parts that are mutually dependent.

\section{Referências}

BAKER, Mona. In other words. Londres: Routledge, 1992.

DESCARTES, René. Discurso sobre o método. Petrópolis: Editora Vozes, 2008.

HOUAISS, Antônio; VILLAR, Mauro de Salles. Dicionário eletrônico Houaiss da língua portuguesa. Rio de Janeiro: Objetiva, 2005.

HUME, David. An Enquiry concerning Human Understanding. Nova York:

Oxford University Press, 1999.

LOCKE, John. Ensaio sobre o entendimento humano. Lisboa: Fundação Calouste Gulbenkian, 1999.

MENÉNDEZ, Marina N. El concepto de equivalencia. In CAGNOLATI, Beatriz E. (Org.). La traductología: miradas para comprender su complejidad. La Plata: Universidad Nacional de La Plata/Facultad de Humanidades y Ciencias de la Educación, 2012. p. 117-187.

PALMER, Robert Frank. Semantics: A new outline. Cambridge: Cambridge University Press, 1976.

REZENDE, Wagner Silveira. A retórica como saber sujeitado. Plural - Revista do Programa de Pós-Graduação em Sociologia da USP, São Paulo, v. 18.2, p. 53-81, 2012.

RÓNAI, Paulo. Escola de tradutores. Rio de Janeiro: Nova Fronteira, 1987. 
SKEAT, William Walter. An etymological dictionary of the English language.

Oxford: Clarendon Press, 1956.

SPENCER, Herbert. The Philosophy of Style. [S.1.]: Project Gutenberg, 2009.

Disponível em: https://www.gutenberg.org. Acesso em 04 jan. 2019. E-book.

SPENCER, Herbert. Essays on education and kindred subjects. Londres: Dent; Nova York: Dutton, 1963.

SPENCER, Herbert. The study of sociology. [Ann Arbor]: University of Michigan Press, 1961.

Referência para citação desta tradução

DOS SANTOS, Thaís. Cinco ensaios de The Philosophy of Style, de Herbert Spencer. Revista PHILIA | Filosofia, Literatura \& Arte, Porto Alegre, volume 1, número 2, p. 596 - 625, outubro de 2019. 